EOmmun: Communication et organisation

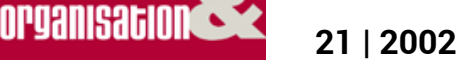

Espace et communication dans les organisations

\title{
Entretien avec Philippe Meurice
}

Marie-Pascale Mignot

\section{OpenEdition}

Journals

Édition électronique

URL : http://journals.openedition.org/communicationorganisation/2700

DOI : 10.4000/communicationorganisation. 2700

ISSN : $1775-3546$

Éditeur

Presses universitaires de Bordeaux

Édition imprimée

Date de publication : 1 mai 2002

ISSN : 1168-5549

Référence électronique

Marie-Pascale Mignot, «Entretien avec Philippe Meurice », Communication et organisation [En ligne],

21 | 2002, mis en ligne le 27 mars 2012, consulté le 10 décembre 2020. URL : http://

journals.openedition.org/communicationorganisation/2700; DOI : https://doi.org/10.4000/

communicationorganisation. 2700

Ce document a été généré automatiquement le 10 décembre 2020.

(c) Presses universitaires de Bordeaux 


\title{
Entretien avec Philippe Meurice
}

\author{
Marie-Pascale Mignot
}

\section{NOTE DE L'ÉDITEUR}

Propos recueillis par Marie-Pascale Mignot

1 Philippe Meurice est architecte et directeur de DEGW France à Paris. Puisant son inspiration dans les nouvelles pratiques de travail et s'appuyant sur son expérience internationale, DEGW France travaille en relation très étroite avec ses clients pour créer des espaces de travail qui stimulent de nouveaux modes d'organisation, et anticipe sur l'évolution des organisations.

2 En 1995, la fusion avec le cabinet de consultants Twynstra conduisit DEGW à élargir son champ de compétences. L'analyse des immeubles intégra les aspects financiers aussi bien que techniques.

3 La conception des espaces de travail et la réalisation des aménagements organisée autour des nouvelles technologies, s'est élargie à la gestion de projet et à l'analyse des process. La gestion du changement dans les espaces de travail permet d'accompagner le management pour franchir cette période de transition.

4 Aujourd'hui, DEGW France (35 architectes et consultants au sein des 250 du Groupe international) développe les services, le conseil, l'aide à la décision, la programmation et le space-planning, l'architecture intérieure, l'architecture et l'urbanisme tertiaire, le management de projet et de construction.

5 A travers toutes ces compétences, DEGW a développé une compétence de la transversalité qui lui permet de garantir l'homogénéité des projets, et permet à ses clients de développer une véritable stratégie pour concevoir un espace de travail global, consistant, qui transcende l'aménagement en projet d'entreprise et stimule la dynamique de l'entreprise.

6 Site internet: www.degw.com 
7 Marie-Pascale Mignot: L 'univers de l'espace de travail semble être particulier, malgré des similitudes avec l'espace domestique sur la question de l' "habité». Pourriez-vous en déterminer les contours, les spécificités et les contraintes?

Philippe Meurice : L'idée d'habiter l'espace est primordiale et on pourrait articuler une vraie réflexion autour de cette notion.

L'habiter domestique répond à la cellule familiale : les fonctions établies culturellement, la structure familiale, et sa représentation sociale sont les deux vecteurs sur lesquels s'organise l'espace domestique. L'espace domestique est le lieu de la famille, c'est le cocon: refuge, lieu de l'affectif mais aussi lieu de représentation sociale au sein de la famille élargie (les cousins, oncles, tantes...) ou vis à vis de la société : les amis, les invités... elle se construit sur des signes de statut, de richesse, de niveau intellectuel... C'est le lieu de la détente, du réconfort, du paraître agréablement dévoilé !

Si l'explosion de l'électroménager a accompagné l'évolution du statut de la femme «au foyer ", la technologie a peu influencé l'espace domestique. Aujourd'hui même, la domotique peine à s'installer dans les maisons malgré la qualité de ses performances.

Le bureau quant à lui est l'espace du travail : rapport à la machine, rapport à la contrainte du travail, à la hiérarchie et à une société plus large que le cercle familial. Pour beaucoup, le lieu de travail est le principal lieu de socialisation. C'est le lieu du conflit, de la lutte pour progresser ou simplement pour préserver son emploi.

Cette caractéristique conflictuelle est au centre même de la définition de l'espace de travail, lieu de tension entre l'individu et l'entreprise: le territoire, le matériel, le mobilier sont-ils ceux de l'individu ou ceux de l'entreprise?

La mission de l'aménageur sera de pousser cette contrainte au plus fort ou de l'atténuer, selon le management qui s'y exerce. L'aménagement de l'espace est signifiant des luttes qui s'y déroulent.

Une nouvelle forme de management veut effacer ce conflit dans son apparence: en travaillant sur une organisation en objectifs et en " soumettant » l'aménagement non plus à des règles d'ordre et de hiérarchie mais à une volonté de confort, en gommant les rapports conflictuels et en faisant une place plus grande à l'individu. L'homme a repris le dessus par rapport au process. Les contraintes sur l'espace glissent du champ fonctionnel « mécanique » au champ sociologique axé principalement sur la notion d'échanges.

Deux grands critères émergent: la satisfaction des besoins individuels qui se traduit notamment par une tendance "cosy" qui emprunte à l'habitat, et la mise en scène des échanges devenus le noyau de la relation des individus dans le travail. Et un nouveau paradoxe en émerge : l'individu est responsable et plus autonome mais c'est le groupe qui permet les interactions nécessaires à l'accomplissement du travail individuel.

Marie-Pascale Mignot: Le bureau conventionnel tend à disparaître et de nouvelles formes de travail esquissent des espaces inattendus. Existe-il plusieurs types d'espaces de travail ?Les avezvous abordés?

Philippe Meurice : Le bureau est avant tout un outil de travail au service du management. Il est donc directement lié aux formes d'organisation du travail et suit l'évolution des modes de travail. Or, sous l'impulsion des nouvelles technologies qui ont provoqué la mondialisation et bouleversé aussi bien les modes de travail que les structures mêmes des entreprises, les lieux de travail se modifient radicalement, fondés sur des postulats très différents 
18 La difficulté réside dans le fait que le monde du tertiaire est très hétérogène. Les entreprises qui le composent sont très diverses par leur activité, leur taille, leur histoire... et de nombreux modes de management existent.

19 Aussi, si on constate l'éclosion et le développement de nouvelles formes d'organisation du travail et de nouveaux concept d'organisation des espaces, les schémas anciens restent valables dans certains cas. L'évolution n'est pas identique pour tous. L'inertie est forte dans certains secteurs.

20 Ceci dit, l'évolution qui mijote depuis quelques années prend maintenant un tour nouveau car on voit apparaître dans le monde du travail des générations qui ont intégré des modes de vie différents correspondant à un monde imprégné des nouvelles technologies.

Le blocage n'est ni technique ni managérial, il est culturel et notamment générationnel.

22 Marie-Pascale Mignot: Quelle place et quelle forme, la communication peut prendre dans le processus de modelage, d'organisation et d aménagement de l'espace?

Philippe Meurice : La communication prise dans un sens complexe, est la pierre angulaire du processus de remodelage. Le difficulté aujourd'hui n'est plus de concevoir des espaces nouveaux. Les expérimentations conduites depuis plusieurs années nous permettent aujourd'hui de disposer d'un panel de solutions bien adaptées aux nouveaux besoins! Aujourd'hui, on ne peut plus vouloir imposer le changement. Il doit être expliqué et intégré par tous. La difficulté est d'accompagner les populations concernées dans ce passage à un autre monde et ceci nécessite une communication forte et complexe. Montrer que le travail a changé, expliquer pourquoi et comment ! C'est l'essentiel du rôle nouveau de l'aménageur car le succès d'une opération dépend de son acceptation par les collaborateurs concernés.

Marie-Pascale Mignot: Quel est votre point de vue de "space-planneur", d'aménageur d'espaces de travail sur les expérimentations menées pat-certaines entreprises d'utilisation de bureaux nomades ou de bureaux partagés... ? Je pense notamment à la société de consultants Andersen à Paris ou l'agence de publicité Chiat Day à New- York.

Aujourd'hui quelles sont les innovations dans l'espace tertiaire qui peuvent transformer nos pratiques.

Philippe Meurice : Comme vous le dites à juste titre il s'agit là d'expérimentations ! Les entreprises concernées ne sont pas représentatives de la majorité des entreprises du tertiaire, donc ces schémas ne peuvent s'appliquer systématiquement à l'ensemble des entreprises. Cependant elles sont fondamentales car elles servent de référence, elles ont permis d'explorer des concepts nouveaux et d'établir de nouveaux jalons, de nouvelles idées qui alimentent la réflexion de tous.

Marie-Pascale Mignot: Souvent on met au banc des accusés les architectes qui conçoivent des espaces de travail perçus plutôt comme une création artistique voir un média pour le Maître d'ouvrage que comme un lien fonctionnel et agréable à vivre pour les utilisateurs.

Y a-t-il quelque chose à remettre en cause ou à mettre à plat dans la relation Maître d'œuvre usagers?

La question de l'usage des espaces souvent traitée par les anthropologues de la communication ou par les sociologues, ne doit-elle pas être traitée en même temps que l'image architecturale?

Philippe Meurice : Il n'y a pas de conflit entre ces notions, il y a simplement nécessité de les hiérarchiser. Nous sommes nous-même une agence d'architecture. Nous sommes 
architectes et notre volonté est d'aménager l'espace pour améliorer la vie de ceux qui le vivent, à l'intérieur comme à l'extérieur. Très vite, dans la pratique nous avons été confrontés au fait que nos seules compétences d'architecte, sur la base de notre formation, ne permettaient pas de répondre efficacement à cet objectif. Le dessin, à la différence du design, met en avant la valeur esthétique, le "paraître » et nous pensions que «l'habiter» comme nous venons de le voir, est plus important. Nous avions découvert également que le « client » a besoin d'être accompagné avant et après le projet proprement dit, qu'il faut l'assister dans sa propre démarche d'émergence de son projet. Or un projet d'aménagement d'espace de travail, c'est un projet d'entreprise.

31 Nous avons alors élargi notre champ de compétences à la programmation, au space planning, à l'urbanisme... sachant que ceci ne peut se concevoir que dans une structure de groupe où ces compétences se complémentarisent. Cette approche remet en cause, dans ce domaine en tout cas, la structure traditionnellement «solaire» du cabinet d'architecte.

Dans la relation Maître d'œuvre et usagers, on peut considérer que la question est celle de l'équilibre entre les valeurs d'usage et la volonté d'image. Jamais pour nous la volonté esthétique, dans les lieux de travail, ne doit contraindre l'usage. L'esthétique ne doit se comprendre que comme l'aboutissement de la maîtrise de l'usage. C'est la définition du design! L'usage est le fondement, le substrat, l'esthétique est un moyen de l'appréhender. En écho à cette volonté, celle de restituer la créativité dans chacun des actes participant à conception de l'espace, à commencer par la pensée sur l'espace. En ce sens l'innovation doit être au cœur de la démarche architecturale avec là aussi une priorité à l'innovation dans les usages: les nouveaux modes de travail, l'évolution des cultures... L'innovation technologique n'est pas une fin en soi, mais sa connaissance nous permet d'appréhender cette évolution des organisations des entreprises, et l'évolution des cultures.

Marie-Pascale Mignot: En terme de conclusion, pensez-vous que la transversalité des disciplines puisse permettre des pratiques professionnelles différentes et apporter une valeur ajoutée à la gestion de l'espace comme enjeu organisationnel ?

Philippe Meurice : Je parlerai même d'une discipline de la transversalité ! L'agence que nous avons voulu mettre en place est fondée sur cette idée de transversalité. Nous avons cherché une réponse au paradoxe entre la volonté d'une démarche globale qui seule garantit l'homogénéité du projet, et la nécessité de spécialisations qui apportent la pertinence des réponses à des problématiques de plus en plus complexes. Ceci signifie que l'agence dispose de professionnels spécialisés dans leur domaine réciproque: programmation, gestion du changement, space planning, conception architecturale, urbanisme*, direction de chantier... et de professionnels qui assurent la cohérence de l'ensemble au sein des projets. La principale force de cette pratique est de pouvoir anticiper, d'assurer la cohérence du projet et d'avoir à tout moment une vision globale et détaillée. La pratique qui en résulte est celle de l'accompagnement du client dans un processus de réflexion, programmation, conception, réalisation qui fonctionne en boucle avec le projet comme point de repère " ponctuel ». Ceci nous conduit, parce que nous sommes dans l'espace de l'entreprise, à travailler sur l'espace vivant, en mouvement. Concevoir en intégrant les activités futures qui remettront en question tous les acquis y compris l'organisation spatiale! L'espace de travail n'est pas un monument, c'est un lieu réactif qui se régénère sans cesse.

* notre champ d'intervention étant les espaces de travail, il s'agit ici de l'urbanisme des parcs d'activités, des «business park» etc.. 